\title{
Synthesis of Nanosized ZSM-5 Zeolites by Different Methods and Their Catalytic Performance in the Alkylation of Naphthalene
}

\author{
Yongchao Wang ${ }^{a}$, O. V. Kikhtyanin ${ }^{b}$, Cheng $\mathrm{Li}^{a}$, Xiaofang Su${ }^{a}$, Xuefeng Bai ${ }^{a}$, and Wei Wu ${ }^{a, *}$ \\ ${ }^{a}$ National Center for International Research on Catalytic Technology, Key Laboratory of Chemical Engineering Process \\ and Technology for High-Efficiency Conversion, College of Heilongjiang Province, \\ School of Chemistry and Material Sciences, Heilongjiang University, Heilongjiang, Harbin, 150080 China \\ ${ }^{b}$ Technopark Kralupy VŠCHT Praha (University of Chemistry and Technology Prague), Praha, 27801 Czech Republic \\ *e-mail: wuwei@hlju.edu.cn
}

Received September 27, 2020; revised October 27, 2020; accepted November 9, 2020

\begin{abstract}
Three nanosized ZSM-5 zeolites were successfully prepared from reactive gels with the same Si/Al ratios by different synthetic procedures that included the use of tetrapropylammonium hydroxide or n-butylamine as a template and a seeding method that did not use an organic additive. The effect of the synthetic method on the physicochemical properties of the prepared samples was investigated by XRD, XRF, XPS, $\mathrm{N}_{2}$ physisorption, SEM, TEM, ${ }^{27} \mathrm{Al}$ MAS NMR, $\mathrm{NH}_{3}$-TPD, and $P y$-FTIR. The catalytic performance of the nanosized ZSM-5 zeolites in the alkylation of naphthalene with methanol was compared. The prepared samples were phase-pure, highly crystalline ZSM-5 zeolites, but they had different bulk and surface $\mathrm{Si} / \mathrm{Al}$ ratios as well as textural and acidic properties. The study of the prepared catalysts in naphthalene methylation revealed that both the acid characteristics of the ZSM-5 nanosized zeolites and their textural properties were responsible for their activity in the reaction. A difference in the composition of monomethylnaphthalenes and dimethylnaphthalenes was attributed to the ability of the catalyst to isomerize the primary reaction products on acid sites located on the external surface of the zeolite crystals. 2,7-DMN was found to be the preferred reaction product over 2,6-DMN when formed at pore entrances to ZSM-5 channels due to the differences in their dimensions. In contrast, 2,6-dimethylnaphthalene could be produced on weaker external Brønsted acid sites, which are hydroxyls attached to octahedral $\mathrm{Al}$ atoms. The presented results show that the method used to synthesize nanoscale ZSM-5 zeolites is a critical factor that determines the physicochemical properties and catalytic performance of the resulting crystals.
\end{abstract}

Keywords: ZSM-5, nanocrystals, naphthalene, alkylation, acidity

DOI: $10.1134 / \mathrm{S} 0965544121030087$

Nanosized ZSM-5 zeolites are widely used in different catalytic applications due to their large external surface area, short channel length, fast diffusion of reactant and product molecules inside the intracrystalline pore volume, and excessive intercrystallite porosity [1-4]. On the other hand, synthetic methods for the preparation of small-sized zeolites inevitably modify the external surface of the obtained crystals, which imparts an outstanding ability to convert large molecules and a high resistance to deactivation by coke and sulfur poisoning, thus further drawing attention to the application of nanosized ZSM-5 zeolites in acid-catalyzed reactions [5].
Usually, the synthesis of small particle-sized ZSM-5 zeolites requires the presence of nitrogen-containing organic molecules as templates. Van Grieken et al. [6] prepared a ZSM-5 zeolite with a crystal size of 10$100 \mathrm{~nm}$ at $170^{\circ} \mathrm{C}$ and $24 \mathrm{~h}$ by using tetrapropylammonium hydroxide as a template. To obtain a smaller nanoscale ZSM-5 zeolite with a crystal size of 10-20 nm, Aguado et al. [7] reduced the crystallization temperature to 70$90^{\circ} \mathrm{C}$ at the expense of a longer crystallization time of 911 days. Zhang et al. [8] prepared a nanoscale ZSM-5 zeolite with a crystal size in the range of $10-500 \mathrm{~nm}$ by a two-step crystallization using $n$-butylamine as a template. All these synthetic methods required using an 
organic compound, and consequently, the calcination of the resulting material for template removal was necessary. Nevertheless, the crystalline framework of small particle-sized ZSM-5 zeolites possesses a large surface tension, as a result, the high temperature might induce the rearrangement of the small crystals to form large aggregates. On the other hand, the use of organic templates is costly, and their burning during zeolite calcination is harmful to the environment. Therefore, attempts to prepare nanosized zeolites without organic additives have been undertaken. Cheng et al. [9] prepared a synthesis gel with a molar composition of $12 \mathrm{Na}_{2} \mathrm{O}$ : $100 \mathrm{SiO}_{2}: 2 \mathrm{Al}_{2} \mathrm{O}_{3}: 2500 \mathrm{H}_{2} \mathrm{O}$, aged it at room temperature for $24 \mathrm{~h}$, and then crystallized at $180^{\circ} \mathrm{C}$ for $24 \mathrm{~h}$. The obtained sample contained well-dispersed ZSM- 5 crystals with a size of $15 \mathrm{~nm}$. Nevertheless, this synthetic method was operative only at the given silica-alumina ratio of $\mathrm{SiO}_{2} / \mathrm{Al}_{2} \mathrm{O}_{3}=50$ with a mordenite admixture at other compositions. The group of Wei Wu reported the synthesis of nanosized ZSM- 5 crystals with $\mathrm{Si} / \mathrm{Al}$ ratios in the range of $30-80$ by a seed-induced method in the absence of an organic template [10]. This method produced wellcrystalline ZSM-5 aggregates of uniform orthogonal nanoparticles with the size of $70-100 \mathrm{~nm}$ and relatively high external surface area. The catalytic performance of the prepared nanosized ZSM-5 zeolites with different $\mathrm{SiO}_{2} / \mathrm{Al}_{2} \mathrm{O}_{3}$ molar ratios was investigated in the alkylation of naphthalene with methanol. Nevertheless, studies on the comparison of the physicochemical properties and catalytic performances of nanosized ZSM-5 zeolites synthesized by different preparation methods are still scarce.

The alkylation of naphthalene (NAPH) with methanol over acidic solids attracts the interest of investigators for several reasons. From a practical point of view, this reaction allows producing 2,6-dimethylnaphthalene (2,6-DMN), which is a key intermediate in the synthesis of polyethylenenaphthalate (PEN) [11-13]. The production and applications of PEN are currently restricted because the multistep industrial synthesis of 2,6-DMN is rather expensive. Thus, the alkylation of naphthalene or methylnaphthalene (MN) with methanol to synthesize 2,6-DMN is an effective route for reducing the cost [14]. However, as many as 10 possible DMN isomers can be formed by the alkylation reaction, and their complete separation, especially 2,6-DMN and 2,7-DMN, is difficult. The main objective of the practical implementation of the one-step method is to develop an efficient catalyst that possesses high activity and stability as well as improved selectivity for the target reaction product, 2,6-DMN. Zeolites varied in structural and acidic properties were used as catalysts for naphthalene alkylation [15-17]. In particular, ZSM-12 zeolites exhibited high activity in the reaction and ensured a high 2,6-/2,7-DMN ratio [15, 16]. Nevertheless, selectivity towards $\beta, \beta$-DMNs did not exceed $50 \%$ with these catalysts. On the other hand, the activity of ZSM-5 zeolites in the reaction was lower, but the selectivity to $\beta, \beta-\mathrm{DMNs}$ was as high as $90 \%$ [18]. Modified ZSM-5 zeolites exhibited reasonable activity in the alkylation of naphthalene or 2-methylnaphthalene and possessed a high 2,6-DMN selectivity [19,20]. Nanosized ZSM-5 zeolites varied by $\mathrm{SiO}_{2} / \mathrm{Al}_{2} \mathrm{O}_{3}$ molar ratios were investigated as catalysts for naphthalene alkylation with methanol [10]. It was concluded that high naphthalene conversion observed for these catalysts was closely associated with their strong acid sites, and high external surface area, while high selectivity to $\beta, \beta-\mathrm{DMNs}$ and $2,6-\mathrm{DMN}$ was attributed to the intermediate acidity, large micropore volume, and the pore mouth size of the nanosized ZSM-5 zeolites. These examples allow suggesting that the structural, textural and acidic properties of zeolites are crucial points for their high activity in naphthalene alkylation and the selective formation of 2,6-DMN. Although Sang et al. [21] examined the differences in the structural and acidic properties of microscale ZSM-5 zeolites synthesized with different templates, a comparative study on the physicochemical properties and catalytic performance of differently prepared nanosized ZSM-5 zeolites have not been reported yet.

In the present work, three nanosized zeolites with ZSM-5 structure and similar $\mathrm{SiO}_{2} / \mathrm{Al}_{2} \mathrm{O}_{3}$ molar ratio were prepared by different synthetic methods. One of the samples was prepared by a seed-induced method, which has the distinctive features of low cost, low pollution, and a possibility to prepare samples in a broad silica-alumina ratio [10]. For comparison, two nanosized ZSM-5 zeolites were synthesized using either tetrapropylammonium hydroxide or $n$-butylamine as templates. The differently prepared ZSM-5 nanocrystals were characterized by physicochemical methods, and their catalytic performance was compared in the alkylation of naphthalene with methanol.

\section{EXPERIMENTAL}

Catalyst preparation. Tetraethyl orthosilicate (TEOS, 98\%, Kermel Chemical Reagent Corporation), 
aluminum isopropoxide (AIP, 98\%, Acros Organics), and tetrapropylammonium hydroxide (TPAOH, 25\%, GuangFu Fine Chemical Research Institute) were used for the preparation of a seed suspension as Si source, $\mathrm{Al}$ source, and template, respectively. The gel with the composition of $1 \mathrm{Al}_{2} \mathrm{O}_{3}: 60 \mathrm{SiO}_{2}: 21.4 \mathrm{TPAOH}: 650 \mathrm{H}_{2} \mathrm{O}$ was crystallized in a Teflon-lined stainless-steel autoclave at $140^{\circ} \mathrm{C}$ for $24 \mathrm{~h}$. The prepared precursor solution was cooled down and used as a seed for subsequent synthesis. In the second synthesis step, silica sol, sodium hydroxide ( $\mathrm{NaOH}$, Sinopharm Chemical Reagent Co., Ltd.), sodium aluminate $\left(\mathrm{NaAlO}_{2}\right.$, Sinopharm Chemical Reagent Co., Ltd.), and $\mathrm{H}_{2} \mathrm{O}$ were mixed with the composition of $12 \mathrm{Na}_{2} \mathrm{O}: 60 \mathrm{SiO}_{2}: 1 \mathrm{Al}_{2} \mathrm{O}_{3}: 2500 \mathrm{H}_{2} \mathrm{O}$. Then the $5 \mathrm{wt} \%$ of the precursor solution was added to the mixture, and the gel was transferred into a Teflon-lined stainlesssteel autoclave and crystallized at $180^{\circ} \mathrm{C}$ for $24 \mathrm{~h}$. A solid product was recovered from the mother liquor by centrifugation and dried at $110^{\circ} \mathrm{C}$ overnight. The residual organic template was removed by calcination in air at $550^{\circ} \mathrm{C}$ for $2 \mathrm{~h}$. The hydrogen form of the sample designated as HNZ-N was obtained by ion exchange with $1.0 \mathrm{~mol} / \mathrm{L} \mathrm{NH}_{4} \mathrm{NO}_{3}$ water solution at $70^{\circ} \mathrm{C}$. This step was repeated twice for $5 \mathrm{~h}$ each, followed by calcination at $500^{\circ} \mathrm{C}$ for $3 \mathrm{~h}$.

The hydrogen forms of nanoscale ZSM-5 zeolites were also synthesized by using tetrapropylammonium hydroxide [6] and $n$-butylamine [22] followed by the ion exchange procedure described above and denoted as HNZ-T and HNZ-A, respectively.

Characterization. X-ray diffraction (XRD) patterns were obtained on a Bruker D8 Advance diffractometer using $\mathrm{Cu} K_{\alpha}$ radiation $(\lambda=1.5405 \AA)$ at $40 \mathrm{kV}$ and $40 \mathrm{~mA}$ in the $2 \theta$ scanning range of $5^{\circ}-35^{\circ}$. The chemical composition of the samples was determined using a Bruker SRS3400 X-Ray Fluorescence (XRF) apparatus at $60 \mathrm{kV}$ and $150 \mathrm{~mA}$. X-ray photoelectron spectroscopy (XPS) measurements were performed on a Kratos AXIS ULTRA DLD using $\mathrm{Al}_{\alpha}(h v=284.6 \mathrm{eV})$ radiation. The nitrogen physisorption isotherms were obtained with a Quantachrome AUTOSORB-1-MP adsorption unit. The samples were outgassed at $300^{\circ} \mathrm{C}$ for $4 \mathrm{~h}$ before adsorption. BET specific surface area was calculated using the Brunauer-Emmett-Teller (BET) equation, and microporous characteristics were obtained from the $t$-plot method. The morphology and the size of ZSM-5 crystals were determined by scanning electron microscopy (SEM) on a Hitachi S-4800 microscope at an accelerating voltage of $30 \mathrm{kV}$. Transmission electron microscopy (TEM) images were recorded on a JEM2100 microscope operating at an accelerating voltage of $200 \mathrm{kV} .{ }^{27} \mathrm{Al}$ MAS NMR spectra were collected using a Bruker Avance-400 spectrometer operating at 104.2 MHz, with a $4 \mathrm{~mm}$ MAS probe. Prior to experiments, $\mathrm{H}$-form samples were exposed to water vapors over $1.0 \mathrm{~mol} / \mathrm{L} \mathrm{NH}_{4} \mathrm{NO}_{3}$ solution overnight. Acidic properties were examined by temperature programmed desorption of ammonia $\left(\mathrm{NH}_{3}\right.$-TPD) on a conventional device equipped with a thermal conductivity detector (TCD). $\mathrm{NH}_{3}$-TPD profiles were obtained in a temperature range from 120 to $650^{\circ} \mathrm{C}$ at a constant heating rate of $10^{\circ} \mathrm{C} / \mathrm{min}$ in a $40 \mathrm{~mL} / \mathrm{min}$ flow of helium. Pyridine-adsorbed Fourier transform infrared ( $P$-FTIR) experiments were carried out on a Perkin Elmer Sp-100 FTIR instrument. Before pyridine adsorption, a wafer was prepared, heated at $350^{\circ} \mathrm{C}$ in a vacuum, and then cooled down to room temperature. Physisorbed pyridine was removed by degassing at $150^{\circ} \mathrm{C}$ for $1 \mathrm{~h}$ in a vacuum and then the FTIR spectra were recorded at room temperature. The concentrations of Brønsted and Lewis acid sites were calculated from the integral intensities of the individual bands characteristic for pyridine adsorbed on Brønsted acid sites (B, $1545 \mathrm{~cm}^{-1}$ ) and on Lewis acid sites (L, $\left.1455 \mathrm{~cm}^{-1}\right)$.

Catalytic testing. Catalytic experiments on naphthalene alkylation were carried out using a fixedbed, continuous-flow microreactor at $400^{\circ} \mathrm{C}$ under $3 \mathrm{MPa}$. The catalyst was activated at $500^{\circ} \mathrm{C}$ for $1 \mathrm{~h}$ under $\mathrm{N}_{2}$ flow of $70 \mathrm{~mL} / \mathrm{min}$ in the constant temperature zone of the reactor and then cooled down to $400^{\circ} \mathrm{C}$. A liquid feed consisting of NAPH, $\mathrm{CH}_{3} \mathrm{OH}$, and 1,2,4-trimethylbenzene $(1,2,4-\mathrm{TMB}$, as a solvent) in a molar ratio of $1: 2: 8$, was pumped into the reaction system simultaneously with a $20 \mathrm{~mL} / \mathrm{min} \mathrm{N}_{2}$ flow. The weight hourly space velocity (WHSV) of the total reaction feed in all experiments was $0.5 \mathrm{~h}^{-1}$. Reaction products were analyzed using an Agilent 6890 GC unit with a flame ionization detector (WCOT PLC capillary column $50 \mathrm{~m} \times 0.25 \mathrm{~mm} \times 0.25 \mu \mathrm{m})$.

\section{RESULTS AND DISCUSSION}

XRD. XRD measurements were carried out to investigate the influence of the different synthetic methods on the phase composition and crystallinity of the prepared materials. The XRD patterns of the three samples (Fig. 1) show diffraction peaks characteristic of a zeolite with MFI topology. No reflection peaks of admixture phases were found, proving the phase purity of the products. The increased width of the diffraction 


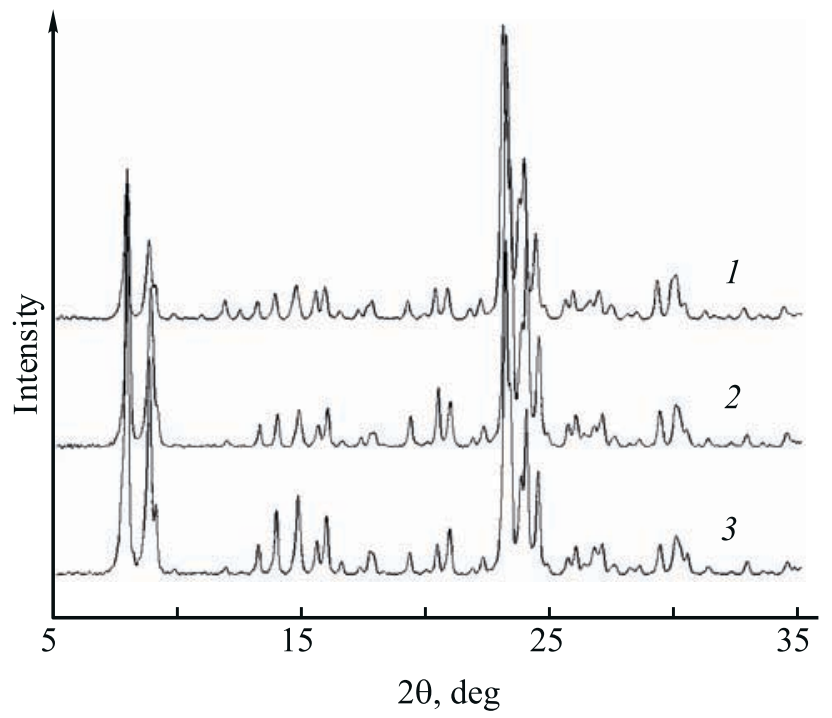

Fig. 1. XRD patterns of nanosized ZSM- 5 zeolites synthesized by different methods: (1) HNZ-T; (2) HNZ-A; (3) HNZ-N.

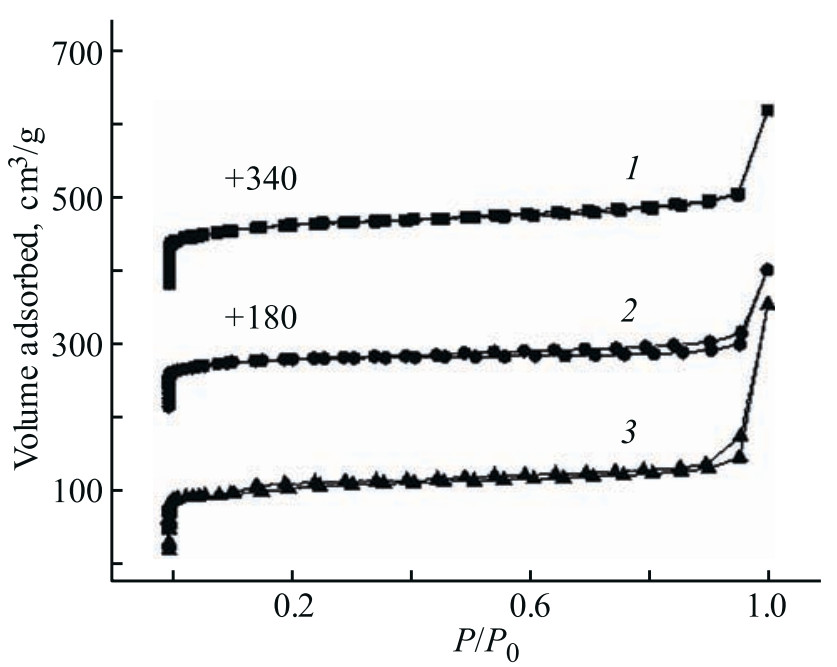

Fig. 2. $N_{2}$ adsorption-desorption isotherms of the nanosized ZSM-5 zeolites synthesized by different methods: (1) HNZ-T; (2) HNZ-A; (3) HNZ-N.

provide information on the distribution of $\mathrm{Al}$ atoms throughout the ZSM-5 framework. Table 1 shows that the $\mathrm{Si} / \mathrm{Al}$ atomic ratio in HNZ-N determined by XPS is higher than that in the bulk composition, indicating that the surface of this zeolite is enriched with Si. In contrast, more $\mathrm{Al}$ atoms are located on the external surface of both the HNZ-T and HNZ-A samples.

$\mathbf{N}_{2}$ adsorption. Figure 2 depicts the $\mathrm{N}_{2}$ adsorptiondesorption isotherms of the prepared ZSM-5 zeolites, which exhibit a typical type I isotherm in by the IUPAC classification.

At low relative pressure $\left(P / P_{0}<0.1\right)$, all of the samples exhibit the high $\mathrm{N}_{2}$ adsorption that is characteristic of a microporous material. At $P / P_{0}>0.4$, a hysteresis loop indicates the presence of secondary mesopores in these materials, which may be caused by the agglomeration of small particles or by surface defects in the ZSM-5 crystalline framework. The $\mathrm{N}_{2}$ adsorption clearly increases at high relative pressure $\left(P / P_{0}>0.9\right)$ due to capillary condensation.

Table 1. Relative crystallinity and $\mathrm{Si} / \mathrm{Al}$ atomic ratio in nanosized ZSM-5 zeolites synthesized by different methods

\begin{tabular}{c|c|c|c|c}
\hline \multirow{2}{*}{ Sample } & \multirow{2}{*}{ Relative crystallinity, $\%$} & \multicolumn{3}{|c}{ Si/Al atomic ratio } \\
\cline { 3 - 5 } & & in reactive gel & bulk $^{\mathrm{a}}$ & on external surface $^{\mathrm{b}}$ \\
\hline HNZ-T & 81 & 30 & 38 & 16 \\
HNZ-A & 100 & 30 & 26 & 15 \\
HNZ-N & 85 & 30 & 20 & 28 \\
\hline
\end{tabular}

${ }^{\text {a }}$ Measured by XRF method.

${ }^{\mathrm{b}}$ Measured by XPS method. 
Table 2. The textural properties of the nanosized ZSM-5 zeolites synthesized by different methods

\begin{tabular}{c|c|c|c|c|c|c}
\hline \multirow{2}{*}{ Samples } & \multicolumn{3}{|c|}{ Surface area, $\mathrm{m}^{2} / \mathrm{g}$} & \multicolumn{3}{c}{ Pore volume, $\mathrm{cm}^{3} / \mathrm{g}$} \\
\cline { 2 - 7 } & BET $^{\mathrm{a}}$ & micropore $^{\mathrm{b}}$ & external & total $^{\mathrm{c}}$ & micropore $^{\mathrm{b}}$ & mesopore \\
\hline HNZ-T & 382 & 308 & 74 & 0.544 & 0.129 & 0.413 \\
HNZ-A & 369 & 343 & 26 & 0.339 & 0.141 & 0.198 \\
HNZ-N & 439 & 366 & 73 & 0.425 & 0.154 & 0.271 \\
\hline
\end{tabular}

${ }^{a}$ BET method.

${ }^{\mathrm{b}} t$-Plot method.

${ }^{c}$ Volume adsorbed at $P / P_{0}=0.99$.

Table 2 summarizes the data for the BET surface area, micropore volume, mesopore volume and external surface area of the prepared samples. HNZ-N exhibits the largest values of BET surface area and micropore volume, which are $439 \mathrm{~m}^{2} / \mathrm{g}$ and $0.154 \mathrm{~cm}^{3} / \mathrm{g}$, respectively. At the same time, HNZ-T possesses the lowest micropore volume but a well-developed mesoporosity, with values of 0.129 and $0.413 \mathrm{~cm}^{3} / \mathrm{g}$, respectively. Both these samples, HNZ-N and HNZ-T, also have high external surface areas of $73-74 \mathrm{~m}^{2} / \mathrm{g}$, which can be attributed to the reduced size of the individual ZSM-5 crystals. In contrast, HNZ-A has the lowest BET surface area and external surface area as well as a small total volume and mesopore pore volume, indicating that the crystal size of this zeolite is larger than that of the other two samples, which is in accordance with the SEM data (see below).

SEM and TEM. SEM images of the samples synthesized by different methods (Fig. 3) show no evidence for the presence of amorphous materials and support the highly crystalline nature of the samples. The polycrystallites of HNZ-T have a cauliflower-like shape and are composed of anomalously small nanosized crystals. The SEM image of HNZ-A exhibits the typical aggregation of cube-shaped, well-dispersed crystals that is consistent with the high relative crystallinity of this sample. The crystals of HNZ-N also have a cube-like shape, but the crystal size is clearly smaller than that of HNZ-A. The different size and shape of the ZSM-5 crystals are mainly due to the different synthetic method, i.e., the influences of the templates and seeding crystals, which introduce a definite texture-directing effect. Note that the obtained SEM images of the prepared ZSM-5 zeolites are in a good accordance with the XRD and $\mathrm{N}_{2}$ physisorption data.

TEM images of the samples (Fig. 4) indicate that the crystals of HNZ-T, HNZ-A and HNZ-N have a cubic-like shape with crystal sizes of 30, 200, and $50 \mathrm{~nm}$, respectively. The crystal size of HNZ-T is smaller compared to that of HNZ-A, but the latter is more dispersed.

${ }^{27}$ Al MAS NMR. The ${ }^{27} \mathrm{Al}$ MAS NMR spectra of the three nanosized ZSM-5 samples (Fig. 5) contain two signals with chemical shifts at $\sim 54$ and $\sim 0 \mathrm{ppm}$. A small signal with a shift at $0 \mathrm{ppm}$ corresponds to octahedral $\mathrm{Al}$ atoms, while an intense signal with a shift at $54 \mathrm{ppm}$ is attributed to isomorphously substituted $\mathrm{Al}$ in a tetrahedral oxygen environment. Table 3 shows the ratio between tetrahedral and octahedral $\mathrm{Al}$ atoms $\left(\mathrm{Al}_{\mathrm{t}} / \mathrm{Al}_{\mathrm{o}}\right)$ in the various nanosized ZSM-5 zeolites. This value is the largest for HNZ-A, indicating that most of the $\mathrm{Al}$ atoms
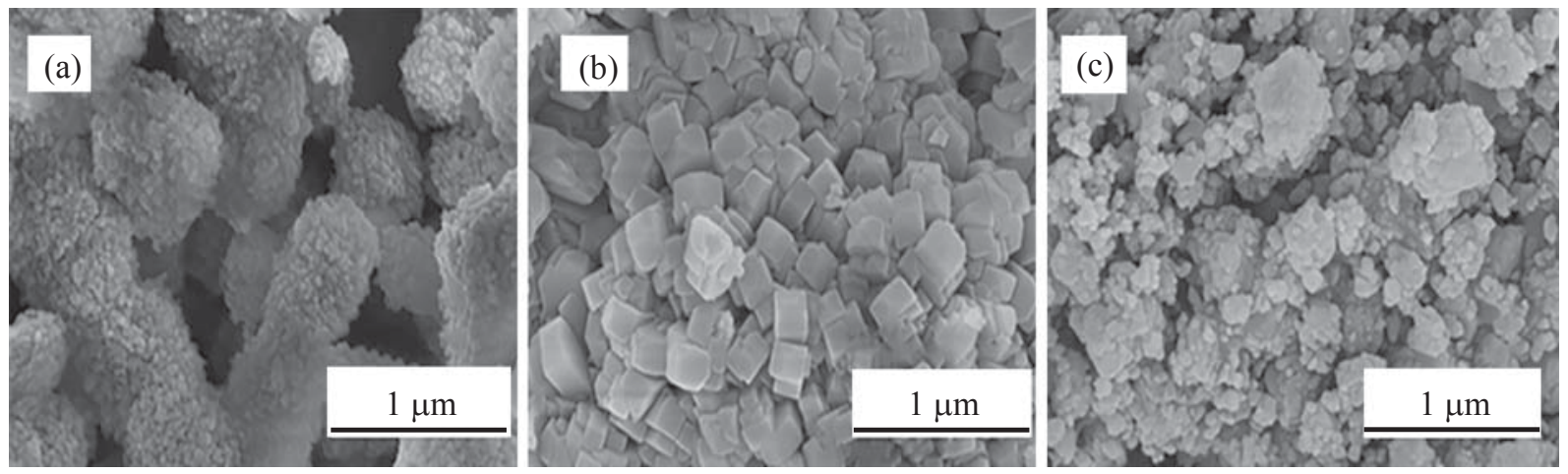

Fig. 3. SEM images of nanosized ZSM-5 zeolites synthesized by different methods: (a) HNZ-T; (b) HNZ-A; (c) HNZ-N. 

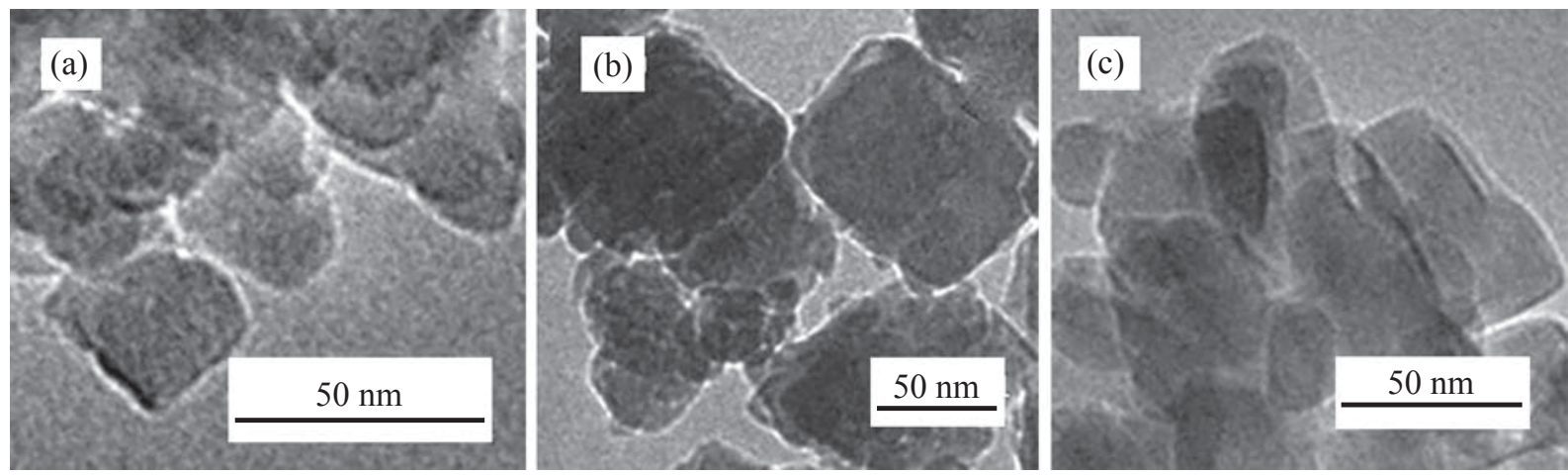

Fig. 4. TEM images of nanosized ZSM-5 zeolites synthesized by different methods: (a) HNZ-T; (b) HNZ-A; (c) HNZ-N.
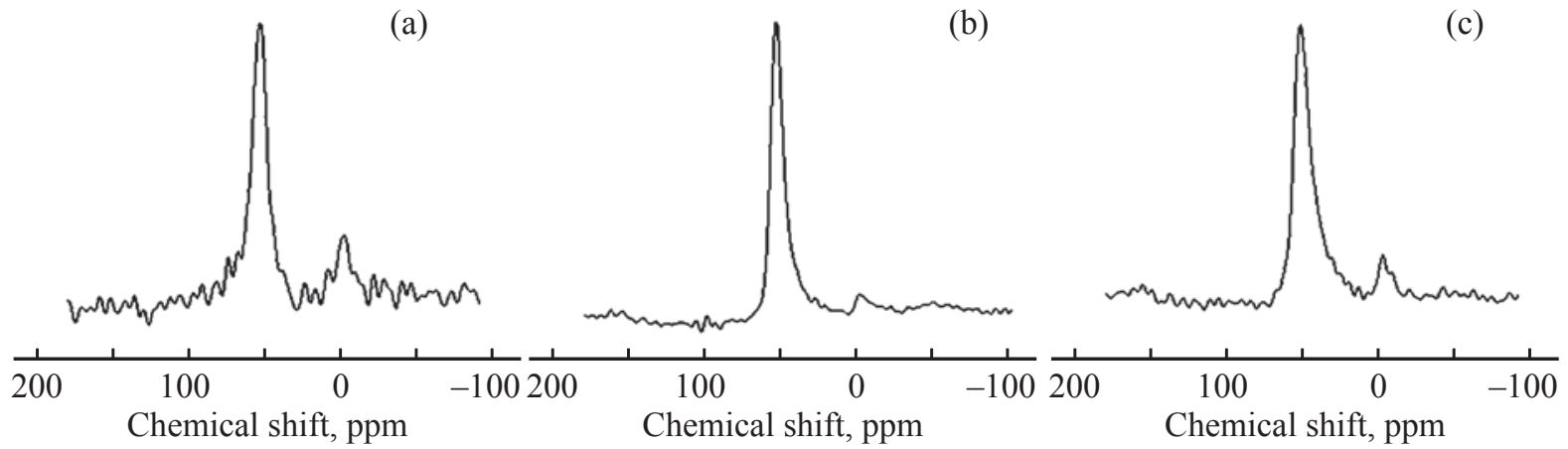

Fig. 5. ${ }^{27}$ Al MAS NMR spectra of nanosized ZSM-5 zeolites synthesized by different methods: (a) HNZ-T; (b) HNZ-A; (c) HNZ-N.

are tetrahedrally coordinated in framework positions, which is in full agreement with the $P y$-FTIR data and is supported by the high crystallinity of this sample. The signals in the NMR spectra of HNZ-T and HNZ-N are broader, suggesting that the $\mathrm{Al}$ atoms in these zeolites have lower average coordination symmetry [24]. Table 3 indicates that the $\mathrm{Al}_{\mathrm{t}} / \mathrm{Al}_{\mathrm{o}}$ ratio is smaller in $\mathrm{HNZ}-\mathrm{T}$ than in HNZ-N, which correlates with a difference in the $\mathrm{Si}$ / Al ratios that were determined by XPS for these samples (Table 1). Nevertheless, it is difficult to unambiguously determine whether the octahedrally coordinated Al atoms in the nanosized ZSM-5 zeolites are completely or partially (hydrolyzed) non-framework, similar to that observed in BEA zeolites [25]. Consequently, the contribution of these sites to the generation of strong acidity, as discussed elsewhere [26-29], is still a matter for debates.

Acidity. Figure 6 depicts the $\mathrm{NH}_{3}$-TPD curves obtained for the three prepared ZSM-5 samples. A high-temperature peak on the presented curves is the characteristic of strong acid sites, while a low-temperature peak is assigned to weak acid sites. HNZ-T has the lowest total density of both weak and strong acid sites which could be concerned with the highest $\mathrm{Si} / \mathrm{Al}$ ratio

Table 3. Acidity and ratio between $\mathrm{Al}$ atoms in tetrahedral and octahedral coordination $\left(\mathrm{Al}_{\mathrm{t}} / \mathrm{Al}_{\mathrm{o}}\right)$ of the nanosized ZSM-5 zeolites synthesized by different methods

\begin{tabular}{c|c|c|c|c|c|c|c}
\hline \multirow{2}{*}{ Sample } & \multicolumn{2}{|c|}{ Temperature of $\mathrm{NH}_{3}$ desorption, ${ }^{\circ} \mathrm{C}$} & \multicolumn{3}{|c|}{ Acidity, $\mathrm{mmol} / \mathrm{g}^{\mathrm{a}}$} & \multirow{2}{*}{$\mathrm{B} / \mathrm{L} \mathrm{ratio}^{\mathrm{b}}$} & \multirow{2}{*}{$\mathrm{Al}_{\mathrm{t}} / \mathrm{Al}_{\mathrm{o}}{ }^{\mathrm{c}}$} \\
\cline { 2 - 6 } & $\mathrm{I}$ & $\mathrm{II}$ & $\mathrm{I}$ & $\mathrm{II}$ & Total & & 4.3 \\
HNZ-T & 199 & 423 & 0.48 & 0.24 & 0.72 & 9.3 & 4.6 \\
HNZ-A & 228 & 429 & 0.54 & 0.61 & 1.15 & 20.0 & 15.3 \\
HNZ-N & 228 & 430 & 0.73 & 0.61 & 1.34 & 13.7 & 10.8 \\
\hline
\end{tabular}

${ }^{a}$ Determined by $\mathrm{NH}_{3}$-TPD data.

${ }^{b}$ Determined by $P y$-FTIR, B/L $=1.5 \times\left(\mathrm{A}_{\mathrm{B}} / \mathrm{A}_{\mathrm{L}}\right)$.

${ }^{\mathrm{c}}$ Determined by ${ }^{27} \mathrm{Al}$ MAS NMR. 


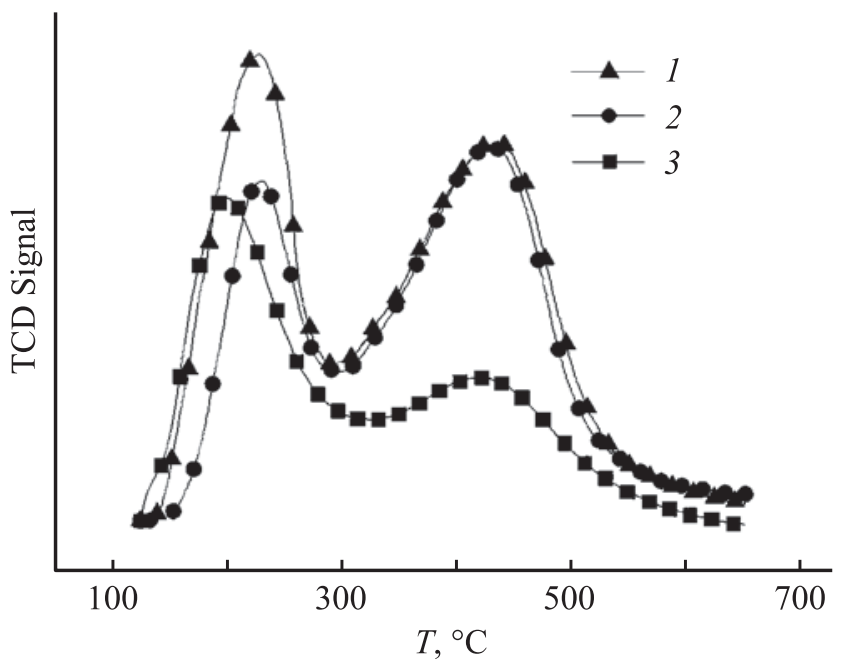

Fig. 6. $\mathrm{NH}_{3}$-TPD profiles of small particle sized ZSM-5 zeolites synthesized by different methods: (1) HNZ-T; (2) HNZ-A; (3) HNZ-N.

in this sample, as determined by XRF. The density of strong acid sites in HNZ-A and HNZ-N is the same, $0.61 \mathrm{mmol} / \mathrm{g}$, which is more than twice as high as that in HNZ-T (Table 3). Nevertheless, HNZ-A and HNZ-N differ in the density of weak acid sites. A difference in the crystallinity (Table 1) and crystal size (Fig. 3) of these samples may be responsible for this difference, assuming that the low-temperature peak in the $\mathrm{NH}_{3}$-TPD curve can be attributed to ammonia desorption from the nonframework $\mathrm{Al}$ or $\mathrm{Si}-\mathrm{OH}$ that are present in the structural defects, according to a previous study [24].

HNZ-A has the largest $\mathrm{B} / \mathrm{L}$ ratio (ratio of Brønsted and Lewis acid sites, determined by adsorption bands appeared at 1545 and $1455 \mathrm{~cm}^{-1}$, respectively [30, 31]) of 20.0, indicating that most of the $\mathrm{Al}$ atoms in this catalyst are in framework positions and, consequently, are

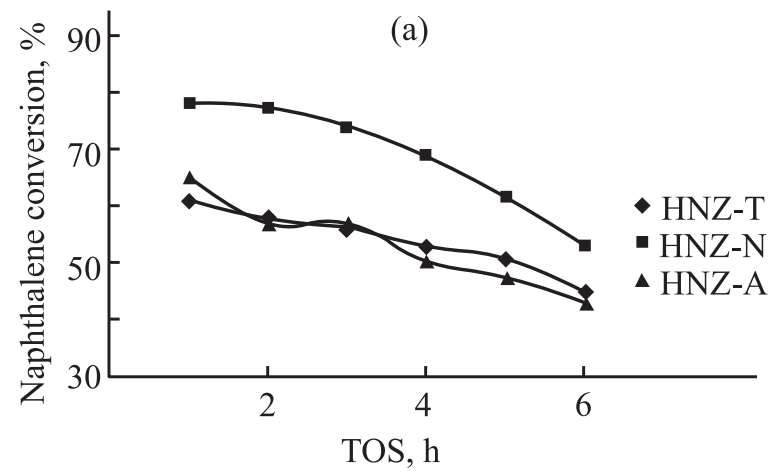

responsible for the ratio between $\mathrm{Al}$ atoms in tetrahedral and octahedral coordination ( $\mathrm{Al}_{\mathrm{t}} / \mathrm{Al}_{\mathrm{o}}$ ratio). Indeed, among the three samples, the HNZ-A has the largest crystal size and the lowest external surface area, therefore, the small amount of Lewis acid sites located on the external surface of the HNZ-A crystals appears reasonable. The B/L ratio in two samples with smaller crystal sizes, HNZ-T and HNZ-N, is significantly lower, with values of 9.3 and 13.7, respectively, which is consistent with the order of $\mathrm{Al}_{\mathrm{t}} / \mathrm{Al}_{\mathrm{o}}$ ratios.

Catalytic activity evaluation. Figure 7 a depicts the change in the naphthalene conversion in dependence on time on stream for the three nanosized ZSM-5 zeolites. The naphthalene conversion observed for the prepared samples at the initial reaction time is in the range $61.0-78.0 \%$, which is substantially larger than that determined in our earlier study on a conventional ZSM-5 zeolite with a similar $\mathrm{SiO}_{2} / \mathrm{Al}_{2} \mathrm{O}_{3}$ ratio under the same reaction conditions [17]. The catalytic performance of all the nanosized ZSM-5 zeolites exhibits a gradual decreasing trend in activity, which is observed in most studies on the alkylation of naphthalene on zeolites with different structural types $[12,19,20]$. This deactivation of the catalyst is usually associated with the formation of polynuclear cracking and coking products, including PMN, at the external surface of the catalyst, thus blocking the access of reactants to the sites at pore mouths [32]. The initial activity of the nanosized ZSM-5 zeolites exhibited the following order: HNZ-N $>$ HNZ-T $\approx$ HNZ-A. Comparison of this trend with the data on the acidity of the ZSM-5 zeolites from Table 3 reveals that the differences in the catalytic activity do not show a clear correlation with the density of strong acid sites. Indeed, the initial naphthalene conversion observed for HNZ-T and HNZ-A is nearly the same and within the range $61.0-64.9 \%$,

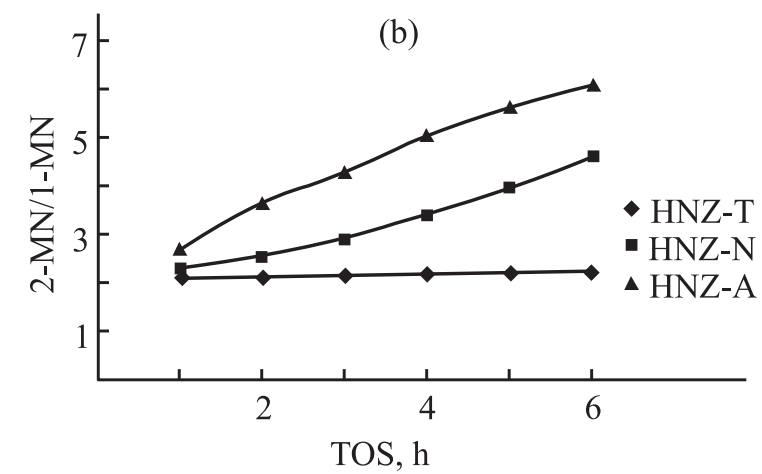

Fig. 7. Catalytic performance of the nanosized HZSM-5 zeolites prepared by different methods. (a) Naphthalene conversion; (b) 2-MN/1-MN ratio. Reaction conditions: $T=400^{\circ} \mathrm{C}, P=3.0 \mathrm{MPa}$, WHSV $=0.5 \mathrm{~h}^{-1}$, NAPH : $\mathrm{CH}_{3} \mathrm{OH}: 1,2,4-\mathrm{TMB}=1: 2: 8(\mathrm{~mol})$. 
whereas the density of strong acid sites in these samples differs markedly, with values of 0.24 and $0.61 \mathrm{mmol} / \mathrm{g}$, respectively (Table 3 ). In contrast, the density of strong acid sites in HNZ-N and HNZ-A is similar with a value of $0.61 \mathrm{mmol} / \mathrm{g}$, but the naphthalene initial conversion is the highest for the former catalyst with a value of $78.0 \%$ compared to $64.9 \%$ for the latter. Apparently, the observed catalytic results cannot be explained exclusively by the acidic sites in the studied zeolites.

The access to the acid sites is inevitably related to the textural characteristics and dimensions of the crystals. The size of the zeolite crystals and their external surface area should be of importance because these factors determine the amount of pore entrances per zeolite weight unit. The average size of the HNZ-A and HNZ-N crystals differ by a factor of 4 with sizes of 50 and $200 \mathrm{~nm}$, respectively, resulting in differences in the textural properties of the samples, the external surface area of HNZ-A is $26 \mathrm{~m}^{2} / \mathrm{g}$, which is significantly lower than the value of $73 \mathrm{~m}^{2} / \mathrm{g}$ obtained for HNZ-N (Table 2). The growth of the external surface in the latter catalyst inevitably increases the amount of pore entrances available for reactant molecules and therefore better facilitates their access to the active sites of HNZ-N compared to HNZ-A. Consequently, HNZ-N exhibits higher activity in the reaction compared to HNZ-A, even if these two zeolites contain the same density of strong acid sites of $0.61 \mathrm{mmol} / \mathrm{g}$ (Table 3 ). Further, HNZ-T has the lowest density of strong acid sites among the studied samples with a value of $0.24 \mathrm{mmol} / \mathrm{g}$, but it also has the smallest crystal size of $30 \mathrm{~nm}$ and a well-developed external surface with a value of $74 \mathrm{~m}^{2} / \mathrm{g}$. As a result, the activity of this zeolite in the alkylation of naphthalene is comparable to the activity of HNZ-A, which possesses a high concentration of acid sites but a small external surface. Thus, a combination of the acid characteristics and textural properties of the ZSM-5 nanosized zeolites is responsible for the observed differences in performance of the catalysts in naphthalene alkylation. In addition, the poor activity of a conventional microsized ZSM-5 zeolite in naphthalene alkylation that was observed under similar reaction conditions also confirms the strong impact of both crystal size and textural properties on the catalytic performance of microporous materials in this reaction.

The repeated alkylation of naphthalene with methanol results in the successive formation of methylnaphthalenes, dimethylnaphthalenes and tri $(+)$ methylnaphthalenes. Figure $7 \mathrm{~b}$ shows the changes in the $2-\mathrm{MN} / 1-\mathrm{MN}$ ratio that were observed during the catalytic experiment for the three nanosized ZSM-5 catalysts. The initial 2-MN/1-MN ratio is in the range 2.08-2.69 and followed the trend HNZ-T > HNZ-N > HNZ-A. The obtained values are slightly higher that at thermodynamic equilibrium (TE), which is 1.55 for the chosen reaction conditions, thus suggesting the preferred formation of 2-MN.

It is believed [33] that the diffusion of methylnaphthalenes in the circular pores of ZSM-5 with sizes of $5.6 \times 5.3$ and $5.5 \times 5.1 \AA$ is strongly hindered. Methylnaphthalenes, which possess a methyl group in the $\beta$-position, have the smallest critical molecular dimensions compared to isomers with a methyl substituent at the $\alpha$-position [20]. It is therefore assumed that methylnaphthalenes with a methyl group only in the $\beta$-position of the naphthalene ring are capable of entering the pore channels of ZSM-5 with difficulty, whereas those with a methyl group in the $\alpha$-position seem completely unable to enter [19]. In contrast, Fraenkel et al. [34] suggested that naphthalene and 2-MN do not actually penetrate the channel system of H-ZSM- 5 but rather react on the catalytic centers located on the zeolite external surface, which are the external Brønsted acid sites located in channel intersection cavities that are characterized by openings larger than 10 rings. Nevertheless, regardless of whether 2-MN is formed inside the pores or in the pore mouth, a 2-MN/1-MN ratio above the TE value suggests the predominant formation of 2-MN under steric constraints followed by the isomerization of the compound on external acid sites, in accordance with a previous report.

With increasing TOS, a different trend in the change in 2-MN/1-MN is observed: after $6 \mathrm{~h}$ of reaction, the ratio reaches 4.8 and 6.0 for HNZ-N and HNZ-A, respectively, indicating a gradual decrease in the isomerization of $2-\mathrm{MN}$ to $1-\mathrm{MN}$ on external centers. For these catalysts, an observed increasing trend in the 2-MH/1-MN ratio with increasing TOS is in line with a decline in naphthalene conversion. Thus, the formation of polynuclear coking products at the entrances of the major channels inhibits access of the reactants to the available sites in the pores and blocks the external acid sites that are responsible for the $\beta \rightarrow \alpha$ isomerization of the methyl group in the naphthalene ring.

In contrast, the 2-MN/1-MNH ratio remains almost constant at a level of 2.1-2.2 during the whole experiment for HNZ-T. The observed decrease in naphthalene conversion with TOS for this catalyst indicates that 
Table 4. Composition of reaction products observed on nanosized ZSM-5 zeolites at naphthalene conversion $\approx 50 \%$. Reaction conditions: $T=400^{\circ} \mathrm{C}, P=3 \mathrm{MPa}$, WHSV $=0.5 \mathrm{~h}^{-1}$, NAPH $: \mathrm{CH}_{3} \mathrm{OH}: 1,2,4-\mathrm{TMB}=1: 2: 8, t=4 \mathrm{~h}$

\begin{tabular}{l|c|c|c|c}
\hline \multicolumn{1}{c|}{ Catalyst } & HNZ-T & HNZ-A & HNZ-N & TE $^{\mathrm{a}}$ \\
\hline Naphthalene conversion, \% & 52.7 & 50.3 & 69.0 & - \\
Mono-MN & Product selectivity, mol \% & & - \\
Di-MN & 58.0 & 58.3 & 56.4 & - \\
Tri-MN & 34.0 & 36.7 & 39.5 & - \\
2-MN/1-MN & 8.0 & 5.0 & 4.1 & 1.55 \\
2,-DMN & 2.2 & 5.0 & 3.4 & \\
2,7-DMN & DMN distribution, \% & & 12.0 \\
2,3-DMN & 20.0 & 26.7 & 23.1 & 11.7 \\
1,7-DMN & 20.3 & 29.8 & 31.0 & 12.1 \\
1,3-DMN & 5.3 & 2.7 & 1.9 & 14.7 \\
1,6-DMN & 18.4 & 16.9 & 18.9 & 14.8 \\
1,4-DMN & 11.1 & 3.6 & 3.3 & 5.0 \\
1,5-DMN & 18.7 & 16.4 & 15.0 & 6.0 \\
1,2-DMN & 1.1 & 0.5 & 1.4 & 9.7 \\
$\beta, \beta-D M N$ selectivity, \% & 3.0 & 1.2 & 3.1 & 35.8 \\
2,6-/2,7-DMN & 2.1 & 2.2 & 2.3 & 1.02 \\
\hline
\end{tabular}

${ }^{\mathrm{a}}$ Thermodynamic equilibrium value (taken from [35]).

blocking of the acid sites at the entrances to major channels with coking products occurs as well; however, the external acid sites of HNZ-T are still able to perform the $\beta \rightarrow \alpha$ isomerization with high efficiency. The physicochemical characterization of the nanosized ZSM-5 zeolites reveals distinguishing features in the properties of HNZ-T. This sample possesses good texture characteristics, i.e., very small crystals with a size of $30 \mathrm{~nm}$ (Fig. 2), and consequently a large external surface and increased mesopore volume with values of $74 \mathrm{~m}^{2} / \mathrm{g}$ and $0.413 \mathrm{~m}^{3} / \mathrm{g}$, respectively (Table 2). Additionally, the ${ }^{27} \mathrm{Al}$ MAS NMR data show that a significant part of the $\mathrm{Al}$ atoms in HNZ-T are octahedrally coordinated with $\mathrm{Al}_{\mathrm{t}} / \mathrm{Al}_{\mathrm{o}}=4.6$ (Table 3 ), while the $P y$-FTIR data suggest that the ratio of the Brønsted and Lewis acid sites of 9.3 is the largest among all of the samples. Taken together, the results prove the presence of a large amount of external acid sites, which are responsible for the $\beta \rightarrow \alpha$ isomerization of the methyl group in the naphthalene ring. In addition, the high mesoporosity of HNZ-T and the small size of its crystals provide fast access of the reactants to the external acid sites and the removal of the reaction products. Consequently, the isomerization of 2-MH to 1-MN on external acid sites is the most effective in the case of HNZ-T compared to the other nanosized zeolites and to a much lesser extent depends on the loss of total activity due to coke formation. On the other hand, HNZ-A, which has poor textural properties, large crystals, and a high $\mathrm{Al}_{\mathrm{t}} / \mathrm{Al}_{\mathrm{o}}$ ratio, exhibits the largest decline in the 2-MN/1-MN ratio with TOS. Accordingly, the obtained results on the content of MNs in the reaction products suggest that the ability of nanosized ZSM- 5 zeolites to carry out the $\beta \rightarrow \alpha$ isomerization of the methyl group in the naphthalene ring is determined by the textural characteristics of the catalysts and their external acidity.

Table 4 presents the total composition of the reaction products obtained from the three nanosized ZSM-5 samples at a naphthalene conversion of $\approx 50 \%$. At a similar conversion level, the relative content of polymethylnaphthalenes (mostly trimethylnaphthalenes) is the largest for HNZ-T, confirming that good textural properties and external acidity contribute to the sequential alkylation of the naphthalene molecule. For all of the catalysts, 2,6-DMN, 2,7-DMN, 1,6-DMN, and 1,7-DMN are the main DMNs isomers and their content is above the TE value, while the content of 1,2-DMN, 2,3-DMN, 1,3-DMN, and 1,4-DMN is below the TE (Table 4). A similar ratio between the content of mono- and bi-ring alkylated compounds was observed in the naphthalene alkylation by SAPO-11 and was explained 

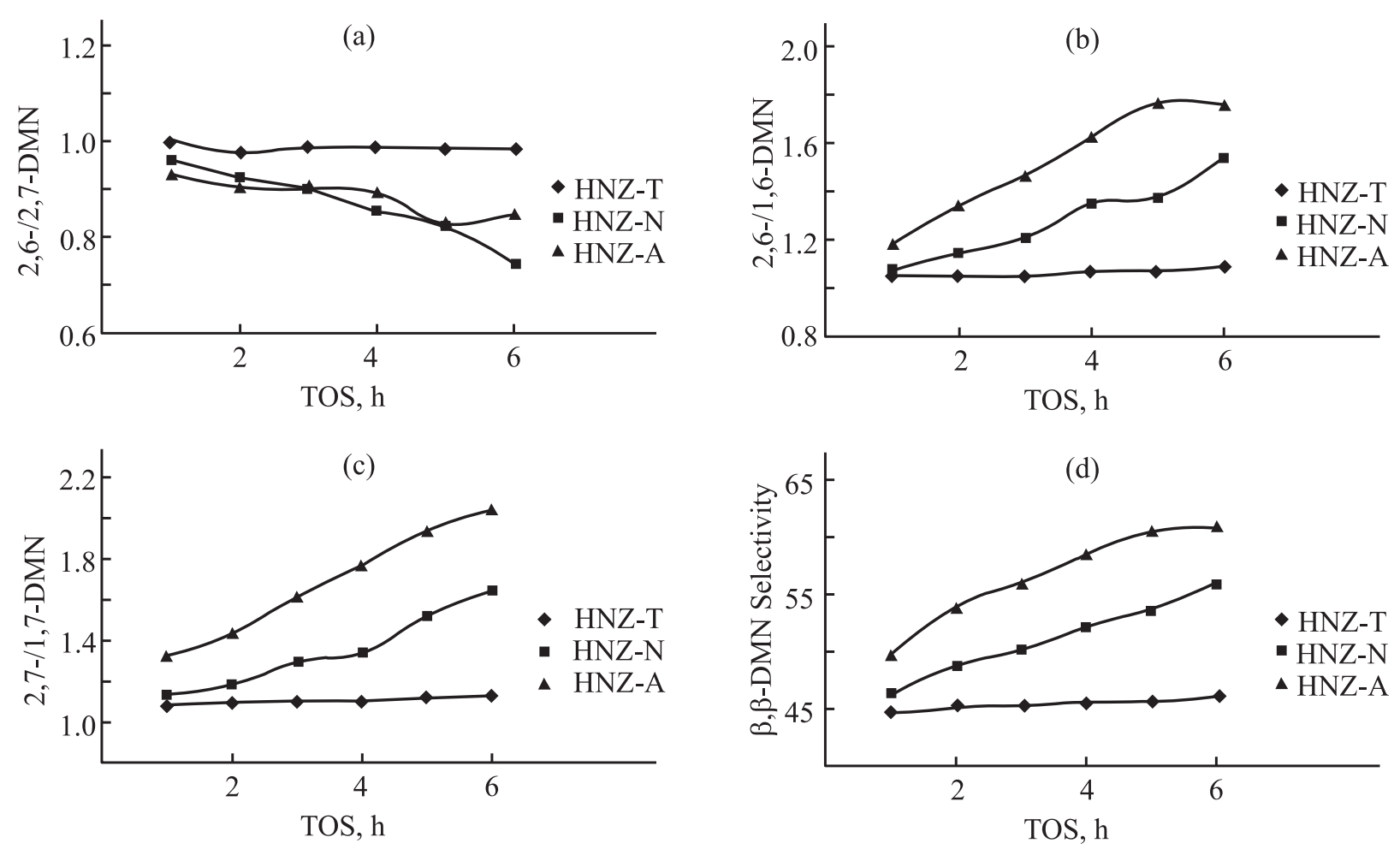

Fig. 8. The change of 2,6-/2,7-DMN (a) as well as 2,6-/1,6-DMN (b), 2,7/1,7-DMN (c) and $\beta, \beta-D M N$ selectivity (d) ratios in dependence on TOS for different nanosized ZSM-5 zeolites.

by the specific orientation of the monoalkylated naphthalene inside the zeolite channels [17]. Similarly, the difference in the content of mono- or bi-ring alkylated dimethylnaphthalenes that was observed for the nanosized ZSM-5 zeolites can also be correlated to a difference in the sizes of naphthalene and methylnaphthalenes: the penetration of a molecule that already bears a methyl group on the naphthalene ring into a ZSM-5 channel is sterically more hindered. Accordingly, the rather high content of 2,3-DMN and 1,3-DMN among the DMNs reaction products that are observed for HNZ-T can be explained by the formation of these compounds on the abundant acidic sites on the external surface.

The value of $\beta, \beta$-DMN selectivity observed for the nanosized ZSM-5 zeolites at a naphthalene conversion of $\approx 50 \%$ is in the range $45.6-59.2 \%$, which is lower than that for a conventional microsized ZSM-5 determined earlier in [17]. The obtained low $\beta, \beta-\mathrm{DMN}$ selectivity of the nanosized ZSM-5 samples suggests the occurrence of secondary transformations of the methylnaphthalenes without any steric restrictions. Indeed, $\alpha, \beta$-isomers such as 1,6-DMN and 1,7-DMN are abundant among the dimethylnaphthalenes.
From the practical point of view, the ratio between the 2,6-DMN and 2,7-DMN isomers is the most important. Table 4 shows that this ratio differs for the three nanosized ZSM-5 zeolites within the range 0.85-0.99, i.e., below the TE value. To determine the factors that affect this ratio, it is necessary to consider the change in the content of isomeric dimethylnaphthalenes during the catalytic experiment.

Figure $8 \mathrm{a}$ shows that for all of the catalysts, the $\beta, \beta-\mathrm{DMN}$ selectivity gradually increases with TOS. This trend is most obvious for HNZ-A because the $\beta, \beta-\mathrm{DMN}$ selectivity for this catalyst rises from $49.8 \%$ at the initial reaction time to $61.0 \%$ at the end of the experiment. The growth in the $\beta, \beta$-DMN selectivity for HNZ-N is moderate with an increase from 46.4 to $56.0 \%$ after $6 \mathrm{~h}$ of TOS, while the $\beta, \beta-\mathrm{DMN}$ selectivity for HNZ-T undergoes only a minor increase from 44.6 to $46.2 \%$. The content of $\beta, \beta$-DMNs reflects the properties of the crystal structure of ZSM-5 since these compounds are predominantly formed on acid sites under steric constraints imposed by ZSM-5 structure. Therefore, the change in the content of $\beta, \beta$-DMNs during the experiment 
is related to the decreasing contribution of their secondary transformations.

In accordance with a previous report [36], isomeric 2,6-DMN and 2,7-DMN are formed by alkylation of $2-\mathrm{MN}$ at either the 6 or 7 position of the naphthalene molecule, respectively. In contrast, the formation of $\alpha, \beta-$ DMNs may occur by two different routes. It is known that isomerization between DMNs is fairly easy if it involves a reversible $\alpha \leftrightarrow \beta$ methyl shift, whereas a shift between the two aromatic rings or between two adjacent $\beta$ positions is significantly more difficult [15]. Therefore, 1,6-DMN and 1,7-DMN can be formed through the isomerization routes 2,6-DMN $\leftrightarrow 1,6-\mathrm{DMN}$ and 2,7-DMN $\leftrightarrow 1,7-\mathrm{DMN}$. Additionally, alkylation of $1-\mathrm{MN}$ at either the 6 or 7 position of the naphthalene molecule also results in either 1,6-DMN or 1,7-DMN. At the beginning of the reaction (Figs. 8b, 8c), the values of 2,6-/1,6-DMN $=$ 0.86 and $2,7-/ 1,7-\mathrm{DMH}=0.80$ are fairly close to the TE values (Table 4). With increasing TOS, these ratios gradually increase for HNZ-N and HNZ-A, but remain approximately constant for HNZ-T, thus correlating with the change in the 2-MN/1-MN ratio. This finding reveals that the increase in the $\beta, \beta-\mathrm{DMN}$ selectivity is related to both a decrease in the 1-MN content and a decrease in the ability of the catalysts to perform the $\alpha \leftrightarrow \beta$ methyl shift. In contrast, the relatively low content of $\beta, \beta$-DMNs, and the high content of $\alpha, \beta$-DMNs in the reaction products on HNZ-T remains approximately constant due to the good isomerizing properties of this catalyst.

For all the catalysts, the initial 2,6-/2,7-DMN ratio is in the range $0.93-1.00$, which is close to the TE value (Table 4). For HNZ-N and HNZ-A, the ratio decreases with TOS along with a decrease in the contribution of the secondary transformations of reaction products. At these reaction conditions, i.e., low isomerization, 2,7-DMN becomes the predominant reaction product from 2-MN alkylation. Pu and Inui [36] suggested that in the absence of external acid sites, the isomerization of 2,7-DMN to 2,6-DMN occurs inside the ZSM-5 channels, which determines the product selectivity. Nevertheless, our results show that the formation of 2,7-DMN is preferred over 2,6-DMN provided that their formation occurs inside the ZSM-5 pores. Jin et al. [20] calculated that 2,6-DMN is somewhat larger than 2,7-DMN, with sizes of $10.06 \times 2.76 \times 6.44$ and $9.73 \times 2.76 \times 6.03 \AA$, respectively. So, 2,6-DMN should experience more diffusion resistance than 2,7-DMN when diffusing from the pore entrances of ZSM-5. Therefore, under the conditions of steric constraints, the formation of 2,7-DMN should be preferred over 2,6-DMN due to the difference in the size of these molecules.

On the other hand, HNZ-T, i.e. the catalyst with a high isomerization ability, produces the ratio of 2,6-/2,7-DMN close to 1 . The possibility of $2,6-\mathrm{DMN} \leftrightarrow 2,7-\mathrm{DMN}$ isomerization inside the zeolite channels or on the external surface of ZSM-5 cannot be completely excluded [36]. However, considering the difficulty of such a step, another possibility for the formation of 2,6-DMN via the participation of external acid sites may be proposed. As discussed previously, HNZ-T has a high content of octahedrally coordinated $\mathrm{Al}$ atoms on the external surface of the zeolite crystals. The hydroxyls on the extraframework or partially framework (defective) aluminum atoms can contribute to the acidity of the zeolite even though they are weaker than Brønsted bridgedhydroxyl acid sites [28]. Thus, the alkylation of 2-MN with methanol could occur via the participation of the hydroxyls attached to octahedral $\mathrm{Al}$ atoms, which behave as Brønsted acid sites. In this case, the formation of 2,6-DMN would be preferred over 2,7-DMN based on frontier molecular orbital theory [20]. Indeed, a higher electron density at C-6 than C-7 in the highest occupied molecular orbital (HOMO) of 2-MN makes position 6 more reactive towards electrophilic substitution than position 7 . Therefore, the alkylation of 2-MN via the participation of less acidic Brønsted acid sites on the external surface of the ZSM-5 crystals should electronically prefer the formation of 2,6-DMN over 2,7-DMN.

\section{CONCLUSIONS}

Three nanosized ZSM-5 zeolites were successfully synthesized from the same synthesis gel composition by either using a template (tetrapropylammonium hydroxide or $n$-butylamine) or employing a seeding method that excluded the addition of an organic template. The prepared samples possessed the same crystalline structure but differed in textural and acidic properties as well as in the distribution of $\mathrm{Al}$ throughout the crystalline framework as a result of the different synthesis methods. The catalytic performances of the nanosized ZSM-5 zeolites were evaluated in naphthalene alkylation with methanol because of its sensitivity to the structural, textural, and acidic properties of zeolitic materials. All of the prepared catalysts possessed a high activity in the alkylation of naphthalene, which was higher than that for a conventional microsized ZSM-5 zeolite. Nevertheless, 
among the nanoscale catalysts, a significant difference in the catalytic performance was observed. Compared to the catalysts synthesized using organic templates, the nanosized ZSM-5 zeolite prepared by a seeding method possessed a larger BET surface area and a higher concentration of acid sites, thus providing the largest naphthalene conversion observed in the study. On the other hand, the ZSM-5 zeolite prepared with tetrapropylammonium hydroxide as a template showed an outstanding isomerization ability, which resulted in 2,6-/1,6-DMN, 2,7-/1,7-DMN, and 2,6-/2,7-DMN molar ratios that were very close to the TE values. In the absence of isomerization of the reaction products on the external surface, both 2,6-DMN and 2,7-DMN can be formed at pore mouths of ZSM-5 channels. Nevertheless, the formation of the latter was more prevalent under the conditions of steric restriction because of the difference in their molecular dimensions. On the other hand, 2,6-DMN can also be formed by the alkylation of 2-MN on the external surface via the participation of weaker acid sites, which are hydroxyls attached to octahedrally coordinated extra-framework or partially framework $\mathrm{Al}$ atoms. In this case, the preferential formation of 2,6-DMN may be due to the higher reactivity of position 6 in the 2-MN molecule compared to position 7. The presented results show that the method used to synthesize nanoscale ZSM-5 zeolites is a critical factor that determines the physicochemical properties and catalytic performance of the resulting crystals. Even when the same chemical composition of a reaction gel is used in the preparation of phase-pure nanosized ZSM-5 zeolites, the physicochemical properties and catalytic performance of the catalysts may differ to a large extent, which is why the optimization of various methods for the synthesis of nanosized zeolites provides wide opportunities for the development and selection of highly active catalysts that possess the necessary catalytic properties.

\section{AUTHOR INFORMATION}

Yongchao Wang, ORCID: http://orcid.org/0000-00016659-7143

Oleg Kikhtyann, ORCID: http://orcid.org/0000-00033951-0182

Cheng Li, ORCID: http://orcid.org/0000-0003-0160-6103

Xiaofang Su, ORCID: http://orcid.org/0000-0001-59721222

Xuefeng Bai, ORCID: http://orcid.org/0000-0002-87577034

Wei Wu, ORCID: http://orcid.org/0000-0001-6984-3581

\section{FUNDING}

This work is supported by the National Natural Science Foundation of China (no. 21676074) International Science and Technology Innovation Cooperation Key Project (2018YFE0108800).

\section{CONFLICT OF INTEREST}

The authors declare that they have no conflicts of interest requiring disclosure in this article.

\section{OPEN ACCESS}

This article is distributed under the terms of the Creative Commons Attribution 4.0 International License (http:// creativecommons.org/licenses/by/4.0/), which permits unrestricted use, distribution, and reproduction in any medium, provided you give appropriate credit to the original author(s) and the source, provide a link to the Creative Commons license, and indicate if changes were made.

\section{REFERENCES}

1. Popov, A.G., Fedosov, D.A., Ivanova, I.I., Vedernikov, O.S., Kleimenov, A.V., Kondrashev, D.O., Miroshkina, V.D., Abrashenkov, P.A., and Kuznetsov, S.E., Petrol. Chem., 2016, vol. 56, p. 237.

https://doi.org/10.1134/S0965544116030117

2. Zhang, L.W., Zhang, H.K., Chen, Z.Q., Ning, Q., Liu, S.Y., Ren, J., Wen, X.D., and Li, Y.W., Catal. Sci. Technol., 2019, vol. 9, p. 7034.

https://doi.org/10.1039/C9CY01672D

3. Ni, Y.M., Zhu, W.L., and Liu, Z.M., ACS Catal., 2019, vol. 9, p. 11398.

https://doi.org/10.1021/acscatal.9b03474

4. Shi, J., Zhao, G.L., Teng, J.W., Wang, Y.D., and Xie, Z.K., Inorg. Chem. Front., 2018, vol. 5, p. 2734.

https://doi.org/10.1039/C8QI00686E

5. Su, X.F., Zan, W., Bai, X.F., Wang, G.L., and Wu, W., Catal. Sci. Technol., 2017, vol. 7, p. 1943.

https://doi.org/10.1039/C7CY00435D

6. Grieken, R. V., Sotelo, J.L., Menéndez, J.M., and Melero, J.A., Micropor. Mesopor. Mater., 2000, vol. 39. p. 135. https://doi.org/10.1016/S1387-1811(00)00190-6

7. Aguado, J., Serrano, D.P., Escola, J.M., and Rodríguez, J.M., Micropor. Mesopor. Mater., 2004, vol. 75, p. 41.

https://doi.org/10.1016/j.micromeso.2004.06.027

8. Zhang, P.Q., Xu, J.G., Wang, X.S., and Guo, H., Chin. J. Catal., 2005, vol. 26, p. 216.

9. Cheng, Y., Wang, L.J., Li, J.S., Yang, Y.C., and Sun, X.Y., Mater. Lett., 2005, vol. 59, p. 3427.

PETROLEUM CHEMISTRY Vol. 61 No. 32021 
https://doi.org/10.1016/j.matlet.2005.06.008

10. Wu, G., Wu, W. Wang, X., Zan, W., Wang, W.J., and Li, C., Micropor. Mesopor. Mater., 2013, vol. 180, p. 187. https://doi.org/10.1016/j.micromeso.2012.11.011

11. Wang, X.X., Liu, Z.M., Wei, X.X., Guo, F., Li, P., and Guo, S.Q., Braz. J. Chem. Eng., 2017, vol. 34, p. 295. https://doi.org/10.1590/0104-6632.20170341s20160120

12. Pu, S.B. and Inui, T., Appl. Catal. A: Gen., 1996, vol. 146, p. 305. https://doi.org/10.1016/S0926-860X(96)00182-2

13. Kim, B.H., Lee, J.G., Yim, T., Kim, H.J., Lee, H.Y., and Kim, Y.G., Tetrahedron Lett., 2006, vol. 47, p. 7727. https://doi.org/10.1016/j.tetlet.2006.08.102

14. Wang, X.X., Zhang, W., and Zhao, L.F., Iran. J. Chem. Chem. Eng., 2015, vol. 34, p. 19.

15. Millini, R., Frigerio, F., Bellussi, G., Pazzuconi, G., and Perego, C., Pollesel, P., Romano, U., J. Catal., 2003, vol. 217 , p. 298. https://doi.org/10.1016/S0021-9517(03)00071-X

16. Wu, W., Wu, W.G., Kikhtyanin, O.V., Li, L.F., Toktarev, A.V., Ayupov, A.B., Khabibulin, J.F., Echevsky, G.V., and Huang, J., Appl. Catal. A: Gen., 2010, vol. 375, p. 279.

https://doi.org/10.1016/j.apcata.2010.01.003

17. Liu, M., Wu, W., Kikhtyanin, O.V., Xiao, L.F., Toktarev, A.V., Wang, G.L., Zhao, A.J., Smirnova, M.Yu., and Echevsky, G.V., Micropor. Mesopor. Mater., 2013, vol. 181, p. 132.

https://doi.org/10.1016/j.micromeso.2013.06.034

18. Jin, L.J., Zhou, X.J., Hu, H.Q., and Ma, B., Catal. Commun., 2008, vol. 10, p. 336.

https://doi.org/10.1016/j.catcom.2008.09.024

19. Zhang, C., Guo, X.W., Wang, Y.N., Wang, X.S., and Song, C.S., Chin. Chem. Lett., 2007, vol. 18, p. 1281. https://doi.org/10.1016/j.cclet.2007.07.025

20. Jin, L.J., Fang, Y.M., and Hu, H.Q., Catal. Commun., 2006, vol. 7, p. 255.

https://doi.org/10.1016/j.catcom.2005.11.012

21. Sang, S.Y., Chang, F.X., Liu, Z.M., He, C.Q., He, Y.L., Xu, L., Catal. Today, 2004, vol. 93-95, p. 729. https://doi.org/10.1016/j.cattod.2004.06.091

22. Wang, K., Dong, M., Niu, X.J., Li, J.F., and Wang, J.G., Cryst. Growth Des., 2018, vol. 18, p. 7548. https://doi.org/10.1021/acs.cgd.8b01354
23. Adnadjević, B., Vukićević, J., Filipović-Rojka, Z., and Marković, V., Zeolites, 1990, vol. 10, p. 699. https://doi.org/10.1016/0144-2449(90)90083-4

24. Kiricsi, I., Flego, C., Pazzuconi, G., Parker, Jr.W.O., Millini, R., Perego, C., and Bellussi, G., J. Phys. Chem., 1994, vol. 98, p. 4627. https://doi.org/10.1021/j100068a024

25. Kotrel, S., Lunsford, J.H., and Knözinger, H., J. Phys. Chem. B, 2001, vol. 105, p. 3917. https://doi.org/10.1021/jp002161v

26. Van Niekerk, M.J., Fletcher, J.C.Q., and O'Connor, C.T., J. Catal., 1992, vol. 138, p. 150. https://doi.org/10.1016/0021-9517(92)90014-9

27. Miller, J.T., Hopkins, P.D., Meyers, B.L., Ray, G.J., Roginski, R.T., Zajac, G.W., and Rosenbaum, N.H., J. Catal., 1992, vol. 138, p. 115. https://doi.org/10.1016/0021-9517(92)90011-6

28. Bjørgen, M., Bonino, F., Kolboe, S., Lillerud, K.P., Zecchina, A., and Bordiga, S., J. Am. Chem. Soc., 2003, vol. 125 , p. 15863 . https://doi.org/10.1021/ja037073d

29. Garralón, G., Corma, A., and Formés, V., Zeolites, 1989, vol. 9, p. 84. https://doi.org/10.1016/0144-2449(89)90015-8

30. Zhang, C., Guo, X.W., Song, C.S., Zhao, S.Q., and Wang, X.S., Catal. Today, 2010, vol. 149, p. 196. https://doi.org/10.1016/j.cattod.2009.04.015

31. Li, Y.Z., Su, X.F., Bai, X.F., Wang, Y.C., Wang, W., Jiang, X., Maximov, A.L., Kolesnichenko, N.V., Bukina, Z.M., and Wu, W., Russ. J. Appl. Chem., 2020, vol. 93, p. 137. https://doi.org/10.1134/S1070427220010152

32. Park, J.N., Wang, J., Hong, S.I., and Lee, C.W., Appl. Catal. A: Gen., 2005, vol. 292, p. 68. https://doi.org/10.1016/j.apcata.2005.05.039

33. Neuber, M., Karge, H.G., and Weitkamp, J., Catal. Today, 1988, vol. 3, p. 11. https://doi.org/10.1016/0920-5861(88)80014-2

34. Fraenkel, D., Cherniavsky, M., Ittah, B., and Levy, M., J. Catal., 1986, vol. 101, p. 273. https://doi.org/10.1016/0021-9517(86)90254-X

35. Stull, D.R., Westrum, Jr.E.F., and Sinke, G.C., J. Chem. Thermodynamics, 1970, vol. 2, p. 153.

36. Komatsu, T., Araki, Y., Namba, S., and Yashima, T., Stud. Surf. Sci. Catal., 1994, vol. 84, p. 1821. https://doi.org/10.1016/S0167-2991(08)63737-4 